ON THE INFILTRATION OF A LIQUID FRCN! IN AN UNSATURATED, FRACIURED POROUS MED M

J. Nitao

T. Buscheck

This Paper was Prepared for Submittal to

Focus ' 89 Nuclear Waste Isolation in

Unsaturated Zones, Las Vegas, Nevada

September 18-21, 1989

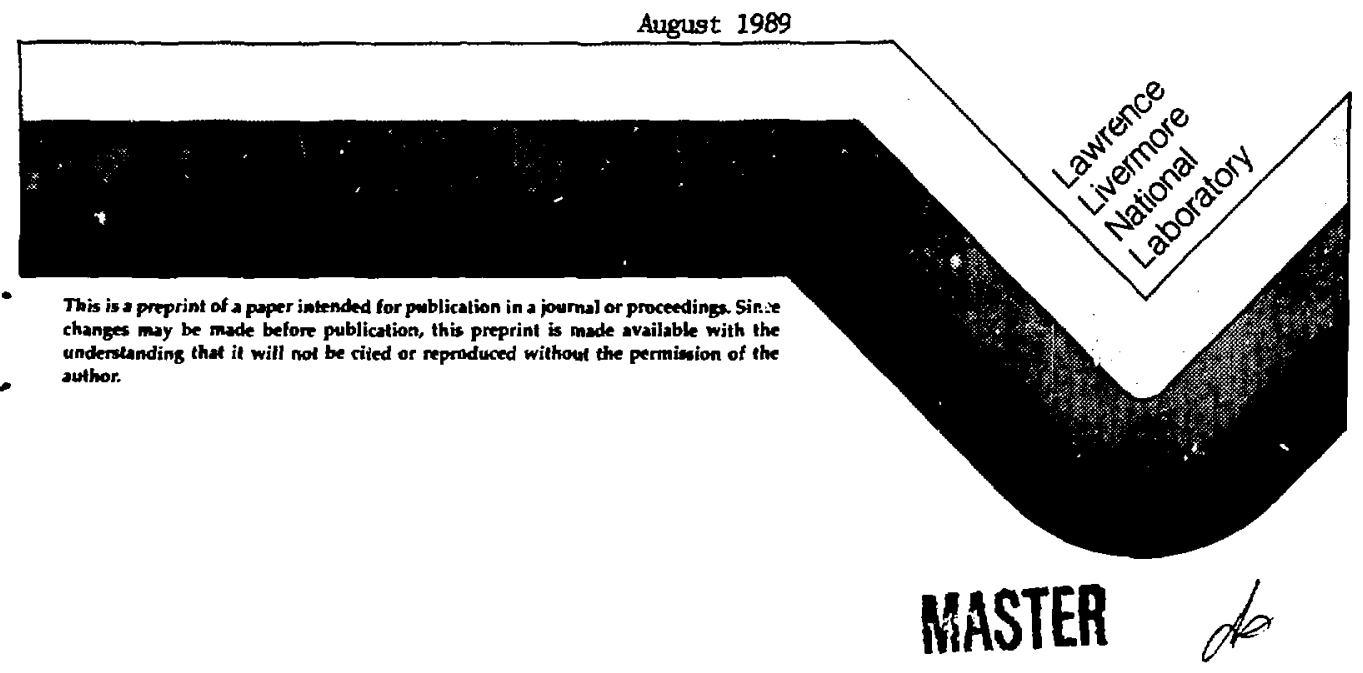

DASTRIBUTION OF THIS BOGLAENT IS UNLMBTITE 


\section{DISCLAIMER}

This document was prepared as an account of work sponsored by an agency of the United States Govemment. Neither the United States Government nor the Untversity of California nor any of their employees. makes any warranty, express or implied, or assumes any legal liablitity or responiblilty for the accuracy, completeness, ol usefulness of any information, apparatus, product, or process disclosed, or represents that Its use would not infringe privately owned rights. Reference hereln to any specific commercial products, process, or service by trade name, trademark, manufacturer, or otherwise, does not necessarly constitute or imply its endorsement, recommendation, or favoring by the United States Government or the University of California. The views and opinions of authors expressed herein do not necessarly state or reflect those of the United States Government or the University of California, and shall not be used for advertising or product endorsement purposes.

Prepared by Yucca Mountain Project (YMP) participants as part of the Civilian Radioactive Waste Management Program. The Yucca Mountain Project is managed by the Waste Management Project Office of the U.S. Department of Energy. Nevada Operations Office. Yucca Mountain Project work is sponsored by the DOE Office of Civillan Radioactive Waste Management. 


\title{
On the Infiltration of a Liquid Front in an Unsaturated, Fractured Porous Medium
}

\author{
Johin J. Nitas and Thomas A. Buscheck \\ Earth Sciences Department \\ Lawrence Livermore National Laboratory
}

This paper is a condensation of the repon "On the Movement of a Liquid Front in an Unsaturated, Fractured Porous Medium, Part 1" that was submitued to YMPO for review on June 16, 1989.

WBS 1.2.2.2

QA Level III

Activity 1-20-]

(This paper was prepared for submittal to the Proceedings of the American Nuclear Society Topical Meeting on "Nuclear Waste Isolation in the Unsaturated Zone". September 38-21, 1989.)

Final Version: December 11, 1989 


\title{
On the Infiltration of a Liquid Front in an Unsaturated, Fractured Porous Medium*
}

\author{
John J. Nitao and Thomas A. Buscheck \\ Earth Sciences Deparment \\ Lawrence Livernore National Laboratory
}

\begin{abstract}
The unsaturated zone at Yucca Mountain, Nevada, is currently under scientific investigation as a proposed site for the permanent storage of high-level nuclear waste. The Topopah Springs unit, in which the proposed reposicory is to be located, as well as most of the other adjacent units, consists primarily of fractured turfeceous rock. A deeper understanding of fracture-macrix interaction is needed for the prediction of water movement around and in the repository. For certain idealized fracture systems that are dominated by fracture flow, we show that the liquid front movement can be classified into physically interpretable, distinctive flow regimes. Asymptotic solutions for the front movement are given for each flow period and comparisons with numerical solutions are made. In addition to applications in nuclear waste storage, the results of our study is relevant to hazardous waste disposal, petroleum recovery, and flow in soil macropores.
\end{abstract}

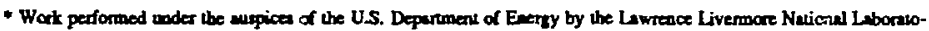
ry under contrat number W-740S-ENG-48. The awtions entefully acknowledge the apport from the Yocca Mountrin Project and the Lawrence Livermore National Labontory Instiortional R \& D Prognm.
} 


\section{Introduction}

The unsaturated zone at Yucca Mountain, Nevada, is a proposed site of the national high-level nuclear waste repository. The various geological units consist primarily of tuffaceous rock with many of the units being highly fractured [Montazer and Wilson, 1984; Klavetter and Peters, 1986]. The mechanics of water infiltration into unsaturated fractured rock is, therefore, of significant practical importunce. In particular, the assessment of waste package periormance and radionuclide transport in the host rock require knowledge of fluid movement in the near-ficld environment. Moreover, the invasion of drilling and mining water ueed during reposilory construction will impact on-site data gathering [Buscheck and Niteo, 1988a], particularly at the exploratory shaft testing facility. Characterizing the repository sile will require the identificution of physical perameters and the andysis of the fundamental processes goveming infiltration and transpont in fracured porous rock.

Understending multiphese fluid processes in fractured porous media is important in other fields of study as well. The secondary recovery of petroleum from naturally fractured reservoirs through water flooding is a prominen example. Our wark is also applicable to heterogeneous unsaturated systems where there is a charp contras in permeability between two types of materials. For example, the flow in a thin layer of high-permeability rock that is sandwiched between two low-permeability layers is also treatable by our analysis. Another area of study related to our work is the flow of water in soil macropores [Beven and Germann, 1982].

The flow of water in 2 real-life fractured rock system is complicated by the complex geometry of the fractures and their spatially varying apertures. In general, the path of water may form sinuous channels, or rivulets, of fluid as it flows through a fracture. In the unsaturated zone, further complications arise from the interaction between the fluid in the fractures and the surrounding matrix. Flows restricted to the matrix may possibly travel across fractures by way of contact pcints [Wang and Narasimhan, 1985]. Before considering these more complicater aspects of fracture flow it would be wise to investigate the simpler problem of flow due to the introduction of liquid at one end of a single fracture. We, therefore, consider a single fracture in an initially unsaturated porous medium intersecting a planar exposed face of the rock mass (Figure 1). Suppose that water is allowed to enter into the 
opening of the fracture with some type of flux or head boundary condition that is uniform across the opening of the fracture. The resulting flow of water in the fracture and the matrix is the focus of our treatment. Note that a sufficient amount of water is assumed to be present at the opening in order to maintain the boundary condition while, at the same time, guaranteeing a continuous slug of water. For example, in the case of constant pressure al the opening equal to a value above ambient conditions, a pond of water must exist at the fracture opening.

Under certain conditions the flux into the fracture may be sufficiantly low that most of the water will be absorbed through matrix imbibition close to the entrance before any significant fracture flow can occur. Movement of the liquid front, if any, in the fracture will be small and will lag behind the front in the marix, leading to what we have termed matrix-dominaled flow. In other cascs, the flux will be sufficiently high such the the fructure flow along the longitudinal direction of the fracture will advance ahead of that in the marrix, a situation we will call fracture-dominated flow. In this latter case, the speed of the front will be governed by an interaction between the driving forces in the fracture and the suction forces in the matrix. Relatively high fluxes are necessary for this case to occur, such as, if there is ponding of water at the entrance to the fracure. A real fracture system existing in the field will have significant spatial variabilities, and it is possible that these different types of flow conditions may occur simultareously at different locations. Future work will also have to consider matrix-dominated flows as well as the transition between the two types of flow.

In our treaument we are interested only in fracture-dominated flow. We treat the idealized problem of one-dimensional How in a planar fracure with uniform aperture. In spile of these simplifications it will be seen that the analysis yields interesting results that may lead, in some cases, to techniques for performing bcunding calculations of water movement for more complicated systems as well as an understanding of some fracture flow processes.

In actual field applications the physical parameters that characterize the flow in a fractured system are often difficult to measure and vary significantly in space. Therefore, their values will have a high degree of uncertainty and variability. Thus, from a practical point of view, what can be realistically achieved is to understand the various physical processes present in the system and, it is hoped, to bound 
the problem. With these goals in mind we have been able, under a class of assumptions, 10 reduce the governing equations inw a single equation of motion describing the movement of the liquid front in the fracture. With this equation we are able to determine the asymptotic behavior of the flow. These solutions are invaluable in revealing various flow processes and flow regimes that may cocur and in determiting the dependency of the flow on various physical parameters.

Most theoretical work in fracture flow has been restricted to saturated conditions and, until recently, relatively little has been done in unsahurated fracture flow. Travis et al. [1984] have presented analytic solutions to the problem of a single slug of finite length traveling down a fracture in an unsaturnted porous medium with the flux into the matrix assumed to be a constant in time. Numerical solutions were given for more realistic time-varying matrix flux condition. Martinez [1988] has also performed numerical calculations for a continuous slug of water and has performed parameter studies applied to Topopeh Spring tuff.

We note here that one of the problems analyzed in our treatment (pressure boundary condition) is mathematically identical to that considered by Philip [1968] who looked at the infiltration process in aggregated medis However, his treatment did not include the effect of gravity on the flow in the macropores (corresponds to frectures, in our study), and, moreover, we are able to show that for fractures there exiss an "intermediate" flow period in addition to the two found by Philip. We also examine the effect of fracture cepillarity on the solutions, and examine the effect of non-equal fracture spacing. Therefore, we believe that much of our work is new. We also mention here that Davidson [1987] has recently considered infiltration from a fracture of finite length that is initially saturated.

Related work has been done in obtaining solutions for contaminant transport in saturated fractured porous media (for example, see [Sudicky and Frind, 1982] and references therein). However, the governing equation for transport in the fracture for those types of problems is the linear convectivediffusive equation. This equation is not adequate for describing the frontal movement of water in an unsalurated fracture. As pointed out by Philip [1968], the equation describing frontal movement is equivalent to a non-linear diffusion equation with a diffusivity that is a delta function centered at unit saturation. 
Another area where theoretical work in multiphase fracture fow has been active is the sxcondary recovery of petroleum reservoirs through water flooding. There, workers have been intercsted in the imbibition of waser into a maturally fractured oil-bearing formation. Van Golf-Racht [1982] summarizes the work in this area. Previous anslyses in the petroletm literature, however, have not given the behavior of the solutions, nor have they elaborated on the various time constants and length scales important to the front movement process.

\section{Assumptions}

We consider the sow resulting from the introduction of a liquid into one end of an inilially dry planar fracture with constant aperture. The flow inside the fracture is treated as a one-dimensional slug with a capillary pressure drop ecross the leading meniscus. The fracture aperture is assumed to be small enough that at each point of the fracture front liquid completely fills the space between the rock walls. The partially saturated rock is assumed to be at uniform initial saturation. In some cases it will be necessary vo assume that the matrix diffusivity for capillary imbibition can be approximated by a constant. We will restrict ourselves to the time span of flow until the slug reaches the end of the fracture. The fracture is assumed to have no intersections with other fractures.

The arrival of the liquid front in the fracture at any given point on the fracture face will rosult in a capillary driven flux into the matrix at that point. The flow field in the matrix as a result of these Iuxes will, in general, be multi-dimensional. However, if the flow in the system is high enough that it is fracture-dominated, as defined earlier, most of the flow lines in the matrix will be primarily orthogona] to the fracture plane. Thus, the flow into the matrix at each point on the fracture can be uncour. pled and treated individually as that of flow into a one-dimensional sub-system. Because the permeability of the matrix is believed to be many orders of magnitude less than the fractures [Klavetter and Peters, 1986, this treatment is applicable to the various tuffaceous units found at Yucca Mountain. This assumption was also used by Travis et al. [1984] and Martinez [1988], and has been confirmed by or numerical simulations which will be presented in a future report. 
Our analysis will not consider the effect of pressure gradients along the length of the fracture upon the imbibition rates into the manix. This effect will be small if the magnitude of the initial suction pressures in the matrix are large relative to the overpressure in the fracture. We will aiso assume that the initial suction forces in the matrix are large enough that for the time span of interest the influence of gravity on the matrix flow (but not on the fracture flow) can be neglected.

In applying the solutions covered in our treatment one must be careful that the boundary conditions are such that the resulting flow does not violate the above assumptions. In many cases the asymptotic solutions can be used to give guidance concerning whether they are satisfied. Future work will have to be done to derive these conditions and confirm them through numerical simulations and, perhaps, Inborabory experiments. An example of when the boundary conditions may be inapprop:iate is in the case of a constant flux boundary condition at the fracture opening. If this fux is too low, one may violate the cordition of fracture-dominated flow, or the slug in the fracture inay be suretehed by gravity and may separate into more than one piece.

\section{The Problem}

\section{Matrix Imbibition Flux}

We now briefly diseuss the form of the imbibition flux into the matrix after pasage of the liquid fracuire frone. The reade is referred o Figure 2 for the coordinace system that is used Suppose that the matrix has a unifom initial saturation distribution. The equations describing the saturation field in the marrix ane

$$
\begin{aligned}
& \downarrow \frac{\partial S}{\partial t}=\nabla \cdot K_{m} k, \nabla \Psi \\
& S(x, y, t=0)=S_{i} \\
& S(x=0, y, t)=S_{m} \quad \text { for } y \leq h(t) \\
& S(x=0, y, t)=S_{i} \quad \text { for } y>h(t)
\end{aligned}
$$

The notation is defined in the nomenclature section at the end of the paper. 
At a given point $y$ for $y \leq h(t)$ the volumetric flux into the matrix along a single face of the fracture is given by

$$
q=-K_{m} \frac{\partial \psi}{\partial x} \quad \text { at } x=0
$$

In general, this flux depends on location, time, and the past history of the liquid fracture front $h(\tau)$ where $\tau \leq t$, that is,

$$
q=q(y, t ; h(\tau), \tau \leq 1)
$$

Under the assumptions dexcribed in the previous section, the imbibition flux $q$ at a point $y$ on the fracture face will depend only on the time when the front first passes by; that is,

$$
\begin{array}{ll}
q(y, t)=0 & t \leq \Omega(y) \\
q(y, t)=q(t-\Omega(y)) & t>\Omega(y)
\end{array}
$$

where $\Omega(y)$ denoles the time when the fracture front first reaches the point $y$. Here, $q(y, t)$ is the matrix imbibition flux into only one fracture wall.

\section{Fracture Flow}

The flow of the liquid in the fracture will be treated as being a slug except with a constant capillary pressure drop at the leading meniscus. The one-dimensional fracture is assumed at any given point to be either completely flled with liquid or completely dry. Let $h(1)$ denote the location of the fracture front with respect to the entrance of the fraclure. We assume that the liquid in the fracture and matrix is incompressible. Let $u(y, t)$ be the liquid velocity at depth $y$ and time $t$ and let $b$ equal to the constant half-aperture of the fracture. From material balance considerations

$$
\frac{\partial u}{\partial y}=-\frac{1}{b} q(y, t)
$$

Now, let $p(y, t)$ be the liquid phase pressure head in the fracture. Assuming Darcy's law for flow in the fracture, we have 


$$
u(y, \ell)=-K_{f}\left(\frac{\partial p}{\partial y}-\beta\right)
$$

where $K_{f}$ is the fracture hydraulic conductivity and $\beta$ is the cosine of the angle of inclination of the fracure from the vertical. The fracture can be oriented either horizontally or inclined downward rela. tive to its opening. The fracure penetration depth $h(t)$ must satisfy the equation

$$
\frac{d h(t)}{d t}=u(h(t), i)
$$

Nove that the function $\Omega(y)$ is related $10 h(t)$ through the relationship

$$
\mathbf{\Omega}(\boldsymbol{h}(\mathbf{t}))=\mathbf{I}
$$

and, hence, is the inverse function of $h(t)$.

We will consider two separate types of bourilary conditions at the entrance to the fracture: pressure head $p_{0}(t)$ and flux $u_{0}(t)$. The pressure head at the leading edge of the front in the fracture is assumed to be a zero datum. Since the equations involve only gradients in head, a non-zero constant capillary drop $-p_{c}$ across the leading edge of the front can be included by adding $p_{c}$ to $p_{0}$. We must, trowever, be careful that the magnitude of the resulting valui of $p_{0}$ is much smalles than the initial suction pressures in the matrix. Otherwise, significant pressure gradients would occur abong the length of the fracture that would couple with the imbibition flux, in violation of one of our basic assumptions. Likewise, the flux boundary condition $u_{0}(t)$ must not be so large that excessive pressures develop in the matrix. It also must not be so small that it can not meet the the flow demanded by the suction and gravily forces in the fracture; otherwise, the slug will become discontinuous violating one of our assumptions. The question of at which critical values of $u_{0}$ will these conditic ss take place will be considered in a later section.

\section{Integro-Differential Equations}

It will be shown $\mathrm{i}_{\mathrm{i}}$ a future report that the above governirg flow equations can, for each of the two types of boundary condition, $\Sigma$ reduced $w$ a single integro-differential equation in $h(t)$. These equations are given as 
Flux-rype boundary condition

$$
\frac{d h(t)}{d t}=u_{0}(t)-\frac{1}{b} \int_{0}^{t} q_{l}(t-s) \frac{d h}{d s} d s
$$

Pressure-rype boundary condition

$$
h(t) \frac{d h(t)}{d t}=X_{f}\left(h(t) \beta+p_{0}(t)\right)-\frac{1}{b} \int_{0}^{1} q_{l}(t-s) h(s) \frac{d h(s)}{d s} d s
$$

where the solution must satisfy the initial condition

$$
h(0)=0
$$

\section{Fracture Geometry}

In our treatment we will consider an infinite array of parallel fractures with the same aperture equal io $2 b$ (sec Figure 3). The spacing between these fractures altemales between distances of $2 a_{1}$ and $2 a_{2}$. The no-flow symmetry lines in the matrix are therefore $a_{1}$ from one side of the fracture and $a_{2}$ from the other. The marix blocks can also aitemate, not only in their size, but also in their material properties, porosity $\phi_{k}$ and diffusivity $\sigma_{k}(k=1,2)$, as well as the initial and maximum saturations $S_{i k}$ and $S_{m k}$.

This geometry includes several special cases, such as the case of a single fracture between two semi-infinite matrix blocks $\left(a_{1}=a_{2}=\infty\right)$, the case of an infinite array of equally spaced fractures $\left(a_{1}=a_{2}\right)$, and the case of two parallel fractures with a finite matrix block in between ( $a_{1}=$ finile, $\left.a_{2}=\infty\right)$.

In the analysis we will assume constant matrix diffusivities. We will show in a future report that for thi case of semi-infinite matrix blocks this assumption is unnecessary and the diffusivity can be a non-constant function of saturation.

\section{Flow Periods}


Depending on whether we have a constant pressure-type or a constant flux-type boundary condition, we can show that the flow in the fracture undergoes various flow regimes, or time periods, with respect to its interaction with the matrix. During each of these periods the function $h(\ell)$, which describes the position of the fronh, can be shown to end asymptotically toward approximate solutions, which on a $\log -\log$ sale form a series of line segments giving the general location of the actual solution curve. But first we wish to introduce some relevant time constants and dimensionless groups. As a convention, we will label the two marrix blocks forming the two sides of the fracture as $k=1$ and $k=2$. Each matrix block can have its own material propertics such as porosity $\phi_{k}$ and effective diffusivity $\sigma_{k}$. (In our notation the diffusivity function is given by $\sigma=\left(K_{m} k_{r} / \phi\right) d \psi / d S$. Here, we will use a constant "effective diffusivity" that will be defined in a repost currently under review.) The initial and maximum saturutions $S_{i k}$ and $S_{m k} c a n$ also be different. The fracture spacing $a_{k}$ was defined in the previous section. From these parameters we define the following relevant time constants Fracture sorativity time constant, the

$$
I_{k k}=\frac{\left[2 b /\left(S_{m k}-S_{i k}\right) \phi_{k}\right]^{2} \pi}{\sigma_{k}}
$$

Average fractwe storativity time constant, Ib

$$
\frac{1}{\sqrt{t_{b}}}=\frac{1}{\sqrt{l_{b 1}}}+\frac{1}{\sqrt{l_{b 2}}}
$$

Fracture interference time constant tak

$$
t_{\varepsilon k}=\frac{a_{k}^{2}}{\sigma_{k}} \pi
$$

A special case of particular interest is when the fractures are uniformly spaced $\left(a_{1}=a_{2}\right)$ and the material properties together with the initial saturation of the two matrix blocks are the same. The parameters that are subscripted with respect to the matrix blocks are not required. From (12) to (14) one has

$$
r_{b}=\frac{\left[b /\left(S_{m}-S_{i}\right) \phi\right]^{2} \pi}{\sigma}
$$




$$
t_{0}=\frac{a^{2}}{\sigma} \pi
$$

We will see later that the solutions can be characterized entirely by the time constants together with the conductivity and fracture orientation. To understand the physical meaning of these time constants, consider a control volume that is a slab of unit thickness that is oriented orthogonal to the fracture. Suppose that imbibition is allowed 10 occur into only one of the matrix blocks. If the saturation along the kength of the imbibition front is taken as being approximately equal to unity, the length given by $L=2 b /\left(S_{m k}-S_{i k}\right) \phi_{k}$, is the distance travelled by the front when the cumulative flux into the matrix block equals the volume of the fracture lying within the slab. The approximate time at which this distance is reached is the fracture storativity time constant $t_{b k}$ given above. If we consider imbibiton into the two matrix blocks simultaneously, $t_{b}$ is the approximate time at which the sum of the two cumulative imbibition fluxes leaving the two walls of the fracture is comparable to the specific fracture volume. Note that $t_{b}$ in (15) does not have the factor of two multiplying $b$ that is present in $t_{b k}$ since each of the two matrix blocks share one-half of the fracture volume. The other time constants $t_{A_{1}}$ are simply the approximate times at which the imbibition front in matrix $k$ reaches the no-flow symmetry line with the respective neighboring fracture. It is interesting to note that although the definition of the time constants assumes a constant or almost constant matrix diffusivity, their definitions in physical tems remain valid even when the diffusivity is a function of saturalion, and are, therefore, applicable even when this assumption does not hold.

We define the following ratios:

Matrix-to-fracture storativity ratio, $\lambda_{k}$

$$
\lambda_{k}=\sqrt{\frac{l_{a k}}{i_{b k}}}=\frac{a_{k}\left(S_{m k}-S_{i k}\right) \phi_{k}}{2 b}
$$

Total storativity ratio, $\lambda$

$$
\lambda=\lambda_{1}+\lambda_{2}
$$


The dimensionless constants $\lambda_{k}$ are the ratios of the initial unsaturated pore volume of the $k$ th matrix to the volume of the fracture while $\lambda$ is the ratio of the total initial pore volume in the matrix to the fracture. When the fracturcs are spaced unifomly and the matrix properties are the same, we have from (17) and (18) that $\lambda$ reduces to

$$
\lambda=\sqrt{\frac{t_{a}}{t_{b}}}=\frac{a\left(S_{m}-S_{i}\right) \phi}{b}
$$

In oder to simplify the discussion, suppose that the matrix blocks on both sides of the fracture have the same flow properties and thas we have a system of paralel fractures with equal spacing. With this assumption we have $t_{a}=t_{a 1}=t_{a 2}$. Analyses which will be given in a future report show that with boundary conditions which do not vary with time, there will generally be three major time periods for the movement of the liquid fracture front. These time periods can be shown to arise from the three sages of matrix imbibition thal can occur at any given point on the fracture face. Let us focus our attention on a single slice of infinitesimal thickess that is arthogonal to the fracture (Figure 4). Suppose that the fracure front bas just reached this slice, and imbibition begins. Stage $A$ for this slice occurs when the cumulative volume of liquid that has imbibed is less than the fracture volume inside the slice. Stage B is when the imbibed volume in the motrix has increased to an amount greater than the frecture volume, but before the matrix front reaches the no-flow symmery boundary of the matrix block due to meighboring fractures. Stage $\mathrm{C}$ occurs after the from reaches the mairix no-flow boundary. The matrix can, therefore, be divided into three zones depending on the stage of imbibition (Figures 5 and 6) with zone 1 corresponding to those points that lie on slices undergoing stage A, zone II corresponds to stage B, and zone III to stage C. These zones propagate with the liquid front as it proceeds into the fracture with zone I occuring near the tip of the fracture, followed by zone II, and, then, by zon: III.

Which time period is occuring depends on which of the zones is the largest. At early times, $1 \leq t_{b}$, most or all of the fracture front lies in zone $I$, and the flow in the fracture is, therefore, influenced only wakly by matrix imbibition and is, insead, dominated by the fracturc boundary 
condition and gravity. As the fracture cront proceeds, a significant part of the matrix is in zone I, i.e. cumulative imbibition fluxes are comparable to the fracture volume, and the front slows down. During this second flow period, $b_{b} \leq t \leq t_{-1}$, there is a balance between (1) mavix suction forces and (2) gravity and, possibly, (3) fracture flow boundary conditions. Finally, as the matrix imbibiticn front appraches the no-flow symmetry planes, the imbibition flux begins to decline, and we enter the third flow period, $t_{e} \leq t$ when most of the matrix is in zone IlI. The front velocily during this flow period approaches a constant.

We are also able to treat other cases: when the matrix blocks do not have the same material and initial properties, and when the fractures are not evenly spaced. In general, we then have $t_{a} \neq t_{a 2}$. In the rest of this work we can assume, without loss of generality, that $t_{a 2} \geq t_{a 1}$. Otherwise, the indices $l$ and 2 are interchanged in what follows.) The only difference from the equal fracture spacing case is that the third flow period is split into two sub-periods IIIa and IIIb because matrix $k=1$ enters flow period III while matrix $k=2$ is still in period II. In particular, there is a period $t_{1} \leq t \leq t_{s 2}$ corresponding to when only matrix block $k=1$ is in flow period III. For later times, $t_{a 2} \leq t$, matrix block $k=2$ is also in flow period II. (These flow periods apply if $f_{1} \leq t_{1} \leq t_{a 2}$, which will be true in most cases. Other less likely orderings of the time constants will lead to other flow periods.)

To summarize, the flow periods are:

Flow period / (boundary and gravity dominased)

$$
t \leq t_{b}
$$

Flow period II. (balanced)

$$
t_{b} \leq t \leq t_{a 1}
$$

Flow period IIla (reduced matrix suction in a single marrix block)

$$
t_{a 1} \leq t \leq t_{02}
$$

Flow period IIIb (reduced matrix suction in both matrix blocks) 


$$
t_{12} \leq t
$$

If a flow period has upper and lower time limits that are comparable or if the upper becornes less than the lower, that particular flow period will not be present For example, when $t_{b}$ is comparable to, or greater than $t_{a}$, , flow period $\Pi$ is non-existent

In some situations, special degenerate cases can occur deris ditg un how the time constants are ordered. For example, suppose that one of the matrix blocks bounding the fracture is much larger than the other but with their diffusivities being equal. That is, $a_{2} \gg a_{1}$ and $\sigma_{1}=\sigma_{2}$. It can then be seen that $t_{e 1} \ll t_{a 2}$. Moreover, suppose that the initial unsalurated pore volume of matrix block $k=1$ is much smaller than the fracture pore volume, which in tum is much smaller than the initial unsaturated pare volume of matrix block $k=2$. We then have the situation where $t_{a} \ll t_{b} \ll t_{a}$. While in How period II the total imbibition flux into block $k=1$ will stan to decline relatively early bccause of the sanall matrix pore volume of this block and and will then go :iw flow period III. This transition will happen before the flux into block $k=2$ has become significant enough to go into flow period Il. The flow in the fracture will revert to being boundary-or grovity-dominaled, and instead of Flow period IIIa we have two periods which we call $I I l a .1$ and $I I a .2$.

Flow period IIJa.I (revent so boundary or gravity dominated)

$$
t_{1} \leq t \leq t_{b 2}
$$

Flow period Iha.2 (partially reduced suction)

$$
t_{b 2} \leq t_{c 2}
$$

In order for this situation to occur we must have $t_{e 1} \leq t_{b 2} \leq t_{a 2}$. It is obvious that there are other orderings of the time constants that can lead to special fow periods not covered by those given here. However, in most situations, such as when both marrix sides of the fracture have nearly identical matrix properties and initial saturations, the three periods we have given in (20) to (23) are the only major ones. The other subcases can be treated, if desired, by using the techniques that will be 
presented in a repart currently under review.

\section{Dimensionless Groups}

Il will be seen later that a convenient definition of ümensioniess ume is obtainet jy taking dime to be relative to the time constant $t_{b}$. Therefore, let us denote by $\tau$ the dimensionless time given by

$$
\tau=t / t_{b}
$$

If will also be convenient to normalize the other time constants relative to $t_{b}$ :

$$
\begin{aligned}
& \tau_{a k}=t_{a k} / t_{b} \\
& \tau_{b k}=i_{b k} / t_{b}
\end{aligned}
$$

The index $k=1,2$ refers to the matrix blorks bounding the fracture. In terms of nomalized lime the flow periods are given as foilows.

Flow period I (fracture flow boundary condition and gravity dominated)

$$
\tau \leq 2
$$

Flow period II (balanced)

$$
1 \leq \tau \leq \tau_{1}
$$

Flow period IIla (reduced matrix suction in a single matrix block)

$$
\tau_{11} \leq \tau \leq \tau_{12}
$$

Flow period IIIb (reduced matrix suction in both marrix blncks)

$$
\tau_{e 2} \leq \tau
$$

The fracture penetration length, $h$, can be made dimensionless $h$ by dividing by $L_{b}$, which is defined to be the distance that would be traveled by the fracture front at time $t=t_{b}$ if no imbibition into the matrix were present. These "imbibition-free" length scales $L_{b}$ can be easily derived for the various combination of boundary conditions and are given in Table I. For example, in the case of 
$p_{0}=0$ with gravity, liquid will travel down the fracture flow at a constant speed equal to $K_{f} \beta$. Thus, at time $t_{b}$ it will have traveled a distance equal $t_{b}=K_{f} \beta t_{b}$. To understand further the meaning of $L_{b}$, note that the gravity and boundary pressure forces including fracture capillarity will dominate over matrix imbibition when the fracture penetration is less than $L_{b}$ since the imbibition into the matrix is relatively unimportant for $t<t_{b}$. In Table I, the last row corresponds to a pressure boundary condition with gravity. There, the value of the length scale $L_{b}$ is computed based on the value computed due to gravity and with the boundary pressure $p_{0}$ set to zero.

In some cases we will also need to define a dimensionless boundary pressure by

$$
\bar{p}_{0}=p_{0} / K_{f} \beta^{2} \sigma_{0}
$$

Recall, that since any capillary head drop $-p_{c}$ across the leading edge of the fracture front can be included into $\rho_{0}$ by adding $p_{c}$, this dimensionless pressure includes the fracture capillary pressure.

\section{Asymptotic Behavior}

\section{Penetration Depth}

In a repon currently under review, we derive the asymptotic solutions to (10) and (11) for the cases where the $\omega_{0}(l)$ or $p_{0}$ boundary conditions are constant in time. The solutions for flow periods I and II bold for mavix diffusivities thas are generel functions of saruration while in the mathematical analysis for flow period III it was necessary to assume that the diffusivity is approximately constant in saturation. In developing a solution for the pressure boundary case, (11), it was convenient for the purposes of exposition to split the problem into (1) the case without gravity (i.e., $\beta=0$ ) and (2) the case with gravily. At early time for the latter case, one must also distinguish whether $p_{0}$ is zero or non-zero relative to ambient head.

In Table II we have summarized the leading terms of the asymptotic expansion for the dimensionless fracture penetration depth $\bar{h}$ for the different types of boundary conditions and for the different flow regimes. The dimensionless variables used here are described in the previous section. Higher order terms can also be derived. Note that all expansions are powers of the dimensionless time $t$. 
When the value of the upper limit of a time period is less than the lower, that particular flow period is not present (eg., if $\tau_{a 1}<1$, then flow period II is not observed, and if $\tau_{a} \leq \tau_{a 1}$, then Illa is not).

The expansions in column 2 of Table II for the pressure boundary condition case with no gravity (i.e., $\beta=0$ ) is a special case of the general expansion given in column 4 and corresponds to the case of a horizontal fracture. Note that during flow period II, for this particular case, the fracture penetration goes as the one-quarter power in time, which is slower than the one-half power movement of the matrix saturation front in the direction longitudinal to the fracture. Hence, the matrix front will eventually overake the fracture front unless flow period III, with its faster one-half power behavior begins sufficiently early. In his theoretical study of aggregated soils Philip [1968] derived asymprotic solutions cquivalent to those in flow periods I and III (column 2 of Table II) but he did not consiôer the intermediate flow period II, probably because this period is not of significant duration for aggregated soils which, because of their relatively small granules, have corresponding time constants with $t_{b}$ comparable $\omega t_{a}$

The fixed pressure boundary condition with $p_{0}=0$ given in column 3 of Table II pertains to the case when the pressure head at the fracture entrance is held at ambient. It is a special case of the solution given in column 4, which includes the general boundary condition in pressure. We have included column 3 in the table because of its simplicily relative to the more general case in column 4 . The situation is complicated in column 4 by the interplay between gravity and the pressure boundary condition. For each of the flow periods in column 4 there are two possible expansions, one pertaining to the time period during which the boundary pressure dominates fracture flow and one for when gravity and matrix imbibition dominates. Each flow period has a dimensionless transition time $\tau^{*}$, which we will define shortly. The applicable expansion is determined by the relationship of $\tau^{*}$ to the upper and lower limits of the respective flow period. If $\tau^{*}$ is less than the lower limit, the second expansion applies over the entire interval. If $\tau^{*}$ is greater than the upper limit, the first expansion is applicable, and if $\tau^{*}$ is between the limits, the first expansion applies for $\tau \leq \tau^{*}$ and the second for $\tau \geq \tau^{*}$. The value of $\tau$ depends on the flow period and is a function of the dimensionless boundary pressure head $\tilde{p}_{0}$ and the matrix-fracture storativity ratio $\lambda$. From Table II we see that it is equal to $\tilde{p}_{0}, \bar{p}_{0}{ }^{2}, \bar{p}_{0}{ }^{2} / \tau_{b 2}, \bar{p}_{0} \lambda$ for 
flow periods I, II, IIIa, and IIIb, respectively. With one exception, the value of $t^{*}$ for a given $\bar{\rho}_{0}$ and $\lambda$ can be shown to lie in unly one of the fiow periods, and, hence, the transition occurs only once. The: exception is if $\tau_{a 2}<\tilde{p}_{0}<\sqrt{\tau_{a 2} \tau_{b 2}}$; in which case, the transition time is in both flow periods lIla and IIIb. Thus, in period IIIa there can be a transition from boundary dominated to matric capillary-gravity, and, then, reversion to boundary dominated at the beginning of period IIIb, and, finally, a setum to matric capillary-gravity dominsted flow.

As we mentioned earlier, if $t_{a 1} \ll t_{b 2} \ll t_{a}$, then flow period IIIa is replaced by IIla.1 and III.2. The expansions for these two subperiods are given in Table III.

The special case, $\tau_{c_{1}}=\tau_{c_{2}}$ and $\tau_{b_{1}}=\tau_{b_{2}}$, is of interest because of its relative mathematical simplicity, and since it includes the equal frecture spacing case with the matrix blocks having identical properties. The expressions in column four of Table II can be reduced to this case by first removing flow period IIIa, changing flow period IIIb to III, and making the following substitutions: $\lambda_{1}=\lambda_{2}=\lambda / 2, \tau_{11}=\tau_{n 2}=\tau_{a}$. Table IV shows the dimensionless fracure penetration for this case with gravity and non-zero boundary pressure conditition. As will be shown later, the dimensionless transition time $\tau^{*}$ from boundary to matric capillary-gravity dominated flew lies in only one of the time periods, and, therefore, the transition occurs only once.

\section{Effects of Fracture Capillarity}

We saw earlier that the boundary condition $p_{0}$ can include the capillary pressure across the leading meniscus of the fracture front by adding the magnitude of the capillary head $p_{c}$ to the entrance pressure. Let us consider the case where the fracture entrance is kept at ambient pressure so that $p_{0}$ is equal to $p_{c}$. For a vertical fracture system $(\beta=0)$, we ask the question: When is fracture capillarity the dorninant driving force? Let us assume that the fractures have equal spacing and identical matrix properties so that the results of Table IV hold. From that table the transition time $\tau^{*}$ from boundary dominated (in this case fracture capillary pressure) to matric capillary-gravity dominated flow is secn to occur in flow period I if $p_{c} / L_{b} \ll 1$, flow period II if $1 \ll p_{c} / L_{b} \ll \lambda$, and now period III if 
$\lambda \ll p_{c} / L_{b}$. Here, we used the fact that $\tau_{a}=\lambda^{2}$ and $p_{0}=p_{0} / L_{b}$. Note that these three conditions on $p_{c}$ are mutually exclusive so that the transition occurs at a single point in time and no reversion to fracture capiliary dominated flow occurs once it starts. It is also interesting to note that the fractuns penetration $h$ at time $\tau=\tau^{*}$ can be shown to be approximately equal to $p_{c}$. Thus, the transition out of the fracture capillarity regime occurs when the hydrostatic head of the liquid in the fracture becomes comparable to the fracture capillary head.

\section{Asymptotic Fracture Penetration Profiles}

It is an interesting fact that the first-order cerms of all of the asymptotic expansions are powers in time $\bar{h} \sim \tau^{*}$ and, when plotted on a log-log scale, will form a series of straight line segments with a corresponding slops $m$ for each flow period. In Figures 7 to 11 we have drawn the generic "penetration profiks" for the three types of boundary conditions that were considered. The line segments are labeled with their respective slopes. For simplicity, we have restricted ourselves in these diagrams to the case when the fracture spacings are equal. Note that in figures 7 and 8 there is a single family of dimensionless curves parameterized with respect to $\lambda$. For figures 9 to 11 the curves are characterized by $\lambda$ and $\tilde{p}_{0}$. Recall that the parameter $\bar{p}_{0}$ is the ratio of the drop in pressure head across the slug (due to boundary head at the entrance and the capillary head at the meniscus) to $\beta L_{b}$ (also recall that $\left.L_{b}=K_{f} \beta t_{b}\right)$. Figure 9 corresponds to the case when $p_{0}<1$. The first line segment on the left corresponds to the flow period during which the pressure drop is small relative to the hydrostatic head.

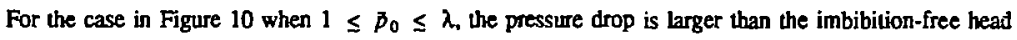
so that the the boundary pressure dominated regime extends past $\tau=1$ into the time period II, $1 \leq \tau \leq \tau_{a}$. Figure 11 corresponds to the case where $\lambda \leq \beta_{0}$ and is boundary pressure dominated until $\tau^{*}=\tilde{p}_{0} \lambda$ in period III, $\tau_{e} \leq \tau$.

These plots have the potential to become useful calculational tools, and are particularly helpful in visualizing the dependence of the asymptotic solutions on the various parameters. 


\section{Parameter Variation}

We now consides the effect of parmeter variation on the fracture penetration for a particular geometry. Consider a vertical fracture with the boundary pressure at the entrance kept at ambient conditions. Such a boundary condition would occur if a shallow pond was present at the entrance. Because of the vertical oxientation, gravity is present. The initial saturation and material propertics of the two matrix blocks bounding the fracture are assumed to be identical. The expressions for the fracture penetration h are listed in Table $\mathrm{V}$ and are dimensional in order to illustrate the dependence on the virious system perameters. It is importunt to note that this table assumes that we stay within a single flow period as the parumeters are variod. In some cases, because of large parameter changes, we may switch into a different flow period. Although these expressions are based on first-order asymptotic approximations, comparisons with numerical calculations indicate that they adequately represcnt the proper parameter sensitivities. We have assumed that the diffusivity $\sigma$ can be approximated as a constant. In order to seperale the dependence of the diffusivity on the porosity and conductivity, we write

$$
\sigma=K_{m} \times 1 \%
$$

The constent $\chi$ is defined here to be some averaged value of $k_{r} d \psi / d S$ where $k_{r}$ is the relative conductivity and $\psi$ is the capillary head as a function of saturation. The time constants can be written as

$$
\begin{aligned}
& t_{b}=\frac{4 \pi b^{2}}{\left(S_{m}-S_{i}\right)^{2} \phi K_{m} \chi} \\
& t_{c}=\frac{\pi a^{2} \phi}{K_{m} \chi}
\end{aligned}
$$

In Table $\mathrm{V}$ we see that $h$ depends linearly on $K_{f}$ for all times. We also see that the $h$ versus $K_{m}$ dependence is significant only during flow period II. However, from (34) and (35), we see that the time constants which define the ranges in the flow periods, $t_{a}$ and $t_{b}$ vary as $K_{m}{ }^{-1}$. During flow period I the fracure penetration $h$ is insensitive to the matrix porosity $\phi$ while it varies as $\phi^{-1 / 2}$ during period II. During period III the sensitivity to becomes even more pronounced, with $h$ varying as $\phi^{-1}$. Regarding the initial matrix saturation $S_{i}$, if the dependence of $\chi$ on $S_{i}$ is weak over some range of values, we 
have that $h$ varies as $\left(S_{m}-S_{i}\right)^{-1}$ during flow periods 11 and Ill while it is insensitive to $S_{i}$ during flow period I. As expecied, only during flow period III is $h$ sensitive to the fracture spacing $a$. However, the time constant $t_{a}$ is very sensitive to $a$, varying as $a^{2}$.

In order to elucidate the dependence of $h$ on the fracture half aperture $b$, let us suppose that the saturated fracture hydraulic conductivity $k$, varies as $b^{2}$ in accordance with some form of the widely used "cubic law" as given by Pouiselle flow theory (see, for example, Huyscom and Pinder [1983]). Thus, the fracture penetration $h$ varies as $b^{2}$ during flow period I and as $b^{3}$ during period II. During period III the fracture penetration $h$ varies as $b^{2}$ if $a \phi\left(S_{m}-S_{l}\right) \ll b$ and as $b^{3}$ if $a \phi\left(S_{m}-S_{i}\right) \gg b$. It is, perhaps, not suprising that $h$ is most strongly affected by the fracture aperture $b$. Note also that the time constant $b_{b}$ is also strongly dependent on fracture aperture, varying as $b^{2}$. Note that the "hydraulic" aperture used in the cubic law need not be equal to the parameter $b$ which is the "volumetric" aperture. In practice, one may take the hydraulic aperture to be smaller than the volumetric aperture.

\section{Comparison with Numerical Solutions}

In this section we give a brief overview of the comparison of the asymptotic solutions with those obuained numerically. More details will be forthcoming in reports still under formal review.

The asymptotic expansions were compared with two separate types of numerical solutions. The first approach involved comparison of the asymptotic solutions with numerical solutions of the integrodifferential equation (11). The first-order asymptotic solutions along with the numerical solution are given in Figure 12 for the case of gravity-driven flow with the fracture entrance kept at ambient pressure. Note that the asymptotic solutions adequately capture the behavior of the solution and except for late times and appear to be conservative upper bounds to the fracture penetration by the liquid front.

The integro-ditferential equation upon which the analytical theory is based was derived under the assumption that the flow in the matrix is orthogonal to the fracture. In actual fracture systems this assumption may not be exactly satisfied. Moreover, under certain conditions, the flow in the fracture may be better represented as that in a porous medjum rather than a slug, as we have assumed. It is 
desirable then to compere our solutions with a two-dimensional simulation not containing these a priori assumptions. Therefore, the second numeical approsch uset in our comparison involves the twodimensional simulation of fracture/matrix flow using a modified version of the TOUGH integrated finite difference code [Preuss and Wang, 1985; Nitao, 1988].

The properties of the fracture used in the two-dimensional simulation are the same as those used by Busckack and Niteo [1988a]. The model represents one out of an infinite set of fractures that are vertical and uniformly spaced. By symmetry, we noed only model half the fracture and the matrix that is on one side; a lateral no-flow boundary is placed down the center of the fracture. and another down the center of the matrix block to represent the symmetry " ne with the neighboring fracture. This fracture is represented by a vertical column of grid blocks with porous media propertics considered to be characieristic of variably saurated flow in a fracture with a nominal aperture of $100 \mu \mathrm{m}$. The absolute permeability of the fracture is besed on the "cubic law" for flow between parallel plates as given by Pouiselle flow theory. The fracture relative permeability curve is estimated by Wang and Narasimhan [1985] based on a simple conceptual model of fracture ilow. The suclion pressure versus saturation curve for the fracture whs extrapolated, using the Young-Loplace equation [Adamson, 1982], from a curve which Wang and Narasimhen [1985] estimaved on the basis of a simple conceptual model of fracture dow. One run was made with this suction curve while another run was made with suction set identically to zero in the fracture to see the effect of fracture capillary. In order to facilitate the incerference beiween neighboring fractures to occur early in time a small fracture half-spacing of $a x$ $2.26 \mathrm{~cm}$ is assumed.

As in Buscheck and Niteo [1988a], the matrix properties, including the characteristic curves which are non-linear, are based on measurements made by Peters und otiers [1984] on sample G4-6 (a sample of Topopah Spring densely welded tiff cored at a depth of 1158 feet within the repository interval at Yucca Mountain). The matrix porosity and initial saturation are taken to be 20 and 65 percent, respectively.

Table VI is a summary of the fracture and matrix propertics used in the two-dimensional model. The first six properties apply to the two-dimensional model. The last two, $a$ and $t_{b}$, are parameters 
required in the comparison with the asymptotic approximation. Recall that $t_{b}$ is the approximate time at which the cumulative matrix imbibition flux is comparable to the specific fracture volume. For early times $t \ll t_{a}$, it can be shown that the first-order asymptotic approximation of the instantaneous speciffe volumetric imbibition flux, $q$, is given in terms of $t_{b}$ and $b$ by

$$
q-\frac{b}{\sqrt{t_{b}}}
$$

Integrating (36) with respect 1 time, we get the cumulative imbibition flux

$$
Q_{m} \sim 2 b \sqrt{t / t_{b}}
$$

By setting $t=t_{b}$ in (37), we find that

$$
Q_{m}-2 b
$$

One practical way of determining $t_{b}$ is as follows. From a one-dimensional matrix imbibition model (where the upstream boundary condition is maintained at 100 percent saturation), the imbibition penctration depth of the saturation front into the matrix, $d_{i m b}$ is plotted against time. The front position can be taken $t o$ be the point where the saturation is equal to the average of the maximum and initial saturations. The time that corresponds to $d_{i m b}=2 b /\left(\phi\left(S_{m}-S_{i}\right)\right)$ is equal to $t_{b}$. The effective matrix diffusivity, $\sigma$, is obtained by applying this value of $t_{b}$ to equation (15). We applied this procedure to our example using the plot of $\log d_{i m b}$ versus $\log t$ found in Buscheck and Nitao [1988a]. Another method would be to plot the cumulative imbibition fiux. The time at which the flux equals $2 b /\left(\phi\left(S_{m}-S_{j}\right)\right)$ is $t_{b}$.

In comparing the plots of $\log \tilde{h}$ versus $\log \tau$ obtained from the first-order asymptotic approximation and the two-dimensional numerical model (Figure 13), we find the two methods agreeing reasonably well. The figure shows two numerical solutions, one with fracture capillary, the other without. The asymptotic solutions given for these two cases are different only for early times $t \ll \tilde{p}_{0}$ when fracture capillarity dominates. Since the two-dimensional numerical model treats the fracture flow characteristics as a porous medium with a saturation-dependent capillary suction curve, it is not 
immediately obvious which value of capillary pressure drop $p_{c}$ w use in the expression for the nondimensional pressure drop term $b_{0}\left(=p_{c} / K_{f} t_{b}\right)$ needed in the asymptotic expansions. In column 4 of Table II we see that the lowest-order contribution of the suction during flow period it is a constant term $2 \tilde{p}_{0} /(\pi-2)$ so that the difference between a simulation with and without fracture capillarity will cnable us to solve for this quantity. This fact was confirmed in our simulations. The value we obtained from this procedure, $\hat{p}_{0} \approx 0.3$, was used in obtaining the asymplotic expansion for $\tau \leq p_{0}$ shown in Figure 13.

During flow period II ( $\tau_{b} \leq \tau \leq \tau_{b}$ ), the asymplotic approximations and the two-dimcnsional model both result in a slope of $m=0.5$. The small roduction in fracture penetration predicted by the two-dimensional model relative to the asymptotic solution is primarily the result of relative permeability effects in the fracture. It appears on the logarithmic plot as an alnost constant downward shift in the two-dimensional model curve. Recall that while the asymptotic solution assumes slug flow in the fracture, the two-dimensional model utilizes a relative permeability curve for fracture flow. The 26 percent reduction in fracture penetration corresponds to a fracture relative permeability, $k_{r}=0.74$. Based on the fracture relative permeability curve, $k_{r}=0.74$ corresponds to a fracture saturation of 95 percent. Accordingly, Buscheck and Nitao [1989)] found that for much of the wetted interval during flow period II, the fracture saturation is close 1095 percent. During flow period III $\left(\tau_{a}<\tau\right)$, the two methods agree very well, with both methods yielding a slope of $m=1$. Because for much of wetted interval of the matrix is fully sarumated, capillary equilibrium between the matrix and fracture (in the two-dimensional model) results in the fracture being fully saturated (corresponding to $k_{r}=1$ ). Consequently, saturation conditions in the fracure (in the two-dimensional model) result in slug flow and there is no reduction in fracture penetration (relative to the asymplotic solution) as was observed during flow period II.

This numerical simulation with its two-dimensional description of matrix flow with gravity and saturation dependent diffusivity function serves to confirm the validity of our basic assumptions underJying our simplified governing equations. Percentage deviation between numerical and approximate analytical solutions is greatest in the transition between flow period 1 and $\mathrm{Il}$ and is about $\mathbf{5 0}$ percent Although using higher-order terms may decrease this figure somewhat, we recall from the previous 
section that the problem is highly sensitive to various parameters such as the Iracture aperture that are difficult to measure and are found to have high variability in the field. Thus, we feel that for most applications it is inappropriate and of dubious value to scek more complex solutions of higher accuracy.

\section{Conclusions}

We have analyzed the various physical processes involved during one-dimensional fracturedominated flow conditions in an unsaturated ponous medium. Such a hydrological condition corresponds most likely to relatively high fluxes such as under ponding conditions at the fracture entrance. For various constant boundary conditions, approximate solutions to the movement of the liquid fracure front were derived. They show that the flow undergoes three major time periods characterized by physically interpretable time constants. The first time constant $t_{b}$ is the time required for the matrix to imbibe a volume equal to that of the fracture starativity. The second is the time $t_{a}$ for the imbibition front to reach the no-flow symmetry line of the neighboring fracture. The first time period occurs when $t<t_{b}$, the second when $t_{b} \ll t<t_{a}$, and the third for $t_{a} \ll t$. Transition periods occur between these main periods. The flow in the matrix can be divided into zones corresponding to the three major fow periods. In many cases it can be shown that, for sufficiently large time, the front in the marrix lags behind the fracture front at a fixed distance. Asymptotic solutions were given which show that the approximate quantilative behavior of the liquid front can be conveniently represented by line segments when plotted on a $\log -\log$ scale. The solutions can be used to understand the dependence on various physical parameters.

Comparison between the asymptotic and numerical solutions confirm the validity of the approach. Our solutions have the potential for estimating the front movement in many practical applications. In addition they present a better quantitative and qualitative understanding of the flow in a fracture in unsaturated porous media These solutions may be helpfu! in the verification of numerical models of fracure flow. 
Further work needs to be done to determine the range of validity of the approximate analytical solutions. We also need to find what new effects are present in two-dimensional fracture flow, and to extend the analytical model, where possible, to fracture nctworks.

\section{Notation}

$a$

$b$

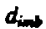

$h$

$\boldsymbol{K}_{\boldsymbol{I}}$

$\boldsymbol{K}_{\boldsymbol{m}}$

$t_{T}$

$L_{b}$

p

$p_{c}$

$\rho_{0}$

$\bar{p}_{0}$

$\boldsymbol{q}$

qI

Q.

s

$\boldsymbol{S}$

$\boldsymbol{S}_{i}$

$S_{m}$

I

$t_{a}$

$t_{b}$

$u$

$u_{0}$

$x$

y

2

$\beta$

$x$

one-half the distance between adjacent perallel fractures

one-half the frocture aperture width

marix imbibition penetration depth

peneiration of the liquid front into the fracture measured from the fracture entrance

frecure-solumated hydraulic conductivity

marix-samated hydraulic conductivity

marix relative permeability function

length that the fracture front would travel during time $t_{b}$ if there were no matrix imbibition

pressure in units of liquid head along the fracture

capillary pressure head at liquid facure front meniscus

pressure in units of liquid bead at the frachure entrance

dimensionaless pressure head at the fracture entrance

specific volumetric flux into the matrix

specific imbibition volumetric flux function into the matrix

cumulative specific volumetric fiux into the matrix

dummy variable of incegration

liquid saturation in the matrix

initial liquid saturation in the matrix

maximum liquid saturation in the matrix limited by air entrainment

ime

fracture interference time constant, approximate time for matrix front to reach

the no-flow boundary

fracture storativity time constant, approximate time for cumulative matrix imbibition flux to become comparable to the volume in the fracture

liquid velocity along the fracture

liquid velocity at the fracture entrance

coordinate distance normal to the fracture

coordinate distance longitudinal to the fracture

flow region length

the cosine of the angle of inclination of the fracture from the vertical

$k, d u / d S$ 
$\lambda$ fracture storativity ratio, the initial unsaturated pore volume of the matrix relative to the volume of the fracture

$\Omega$ function $\Omega(y)$ denoting the time at which the fracture front first reaches point $y$

$\phi \quad$ matrix porosity

$\Psi \quad$ matrix capillary pressure head

o matrix diffusivity, or effective matrix diffusivity

$\tau \quad$ dimensionless time equal to $t / t_{b}$

$\tau_{e} \quad t_{a} / t_{b}$

$\tau_{b k} \quad t_{b k} / t_{b}, k=1,2$

$\tau^{*} \quad$ dimensionless transition time from boundary dominated flow to flow dominated by gravity and matrix capilary forces

\section{References}

Adamson, Arhur. W., Physical Chemistry of Surfaces, John Wiley and Sons, 4th ed., 1982.

Beven, K. and P. Germann, Macropores and Water Flow in Soils, Waler Resources Research, vol. 18, no. $5,1311-1325,1982$.

Buscheck, T.A. and JJ. Nitro, Estimates of the Hydrologic Impact of Drilling Water on Core Samples taken from Partially Saturated Densely Welded Tuff, Lawrence Livermore National Laboratory, UCID-21294, 1988a.

Buscheck, T.A. and JJ. Nitao, Estimates of the Width of the Weting Zone Along a Fracture Subjected to an Episodic Infillration Event in Variably Satwated, Densely Welded Tuff, Lawrence Livermore National Laboratory, UCID-21579, 1988b.

Davidson, M. R., Asymptotic Infiltration into a Soil which Contains Cracks or Holes but whose Surface is Otherwise Impermeable, Transport in Porous Media, vol. 2, 165-176, 1987.

Huyacom, P.S. and G.F. Pinder, Computational Methods in Subsurface Flow, Academic Press, 1983.

Klavetler, E.A., and R.R. Peters, Fluid Flow in a Fractured Rock Mass, Sandia National Laboratories, SAND85-0855, 1986.

Martinez, MJ., Capillary-Driven Flow in a Fracture Located in a Porous Medium. Sandia National Laboratories, SAND84-1697, 1988.

Montazer, P. and W.E. Wilsan, Conceptual Hydrologic Model of Flow in the Unsaturated Zone, Yucca Mountair, Nevada, U.S. Geological Survey, Water-Resources Investigations Repon 84-4345, 1984.

Nitao, J.J., Numerical Modelling of the Thermal and Hydrological Environment around a Nuclear Waste Package Using the Equivalent Continum Approximation: Horizontal Emplacement, Lawrence 
Livermore National Laboralory, UCID-21444, 1988.

Peters, R.R., E.A. Klavetter, I.J. Hall, S.C. Btair, P.R. Heller, and G.W. Gee, Fracture and Matrix Hydrologic Characteristics of Tuffaceous Materials from Yucca Mountain, Nye County, Nevada, Sandia National Laboratories report no. SAND84-1471, 1984.

Philip, J.R., The Theory of Absorption in Aggregated Media, Australian Journal of Soil Research, vol. 6, $1-19,1968$.

Pruess, K. and J.S.Y. Wang, TOUGH - A Numerical Model fir Nonisothermal Unsaturated Flow to Study Waste Cannister Heating Effects, Mat. Res. Soc. Symp. Proc., vol. 26, Elsevier Science, 1984.

Sudicky, E.A., and E.O. Frind, Contaminant Transport in Fractured Porous Media: Analytical Solutions for a System of Parallel Fractures, Water Resources Research, vol. 18, no. 6, 1634-1642, (1982).

Travis, B.J., S.W. Hodson, H.E. Nuttall, T.L. Cook, and R.S. Rundberg, Preliminary Estimates of Water Flow and Radionuclide Transport in Yucco Mountain, Los Alamos National Laboratory. LA.UR. 84-40, 1984.

van Golf-Racht, T.D., Fundamentals of Fractured Reservoir Engineering. Elsevier, 1982.

Wang, J.S.Y. and T.N. Narasimhan, Hydrologic Mechanisms Governing Fluid Flow in a Partially Sa. twrated, Fractured, Porous Medium, Waler Resources Research, vol. 21, no. 12, 1861-1874, 1985. 
FIGURES 


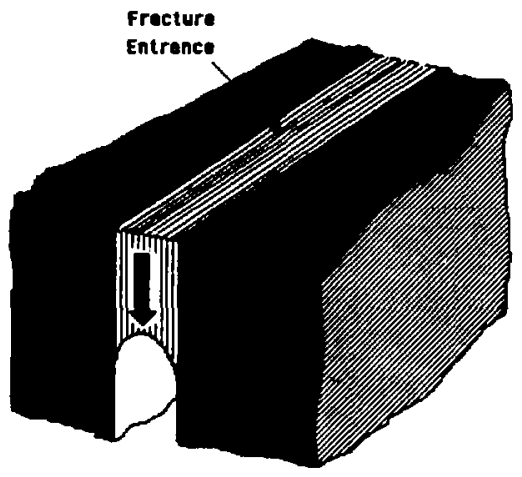

Figure 1. Idealized Fracture

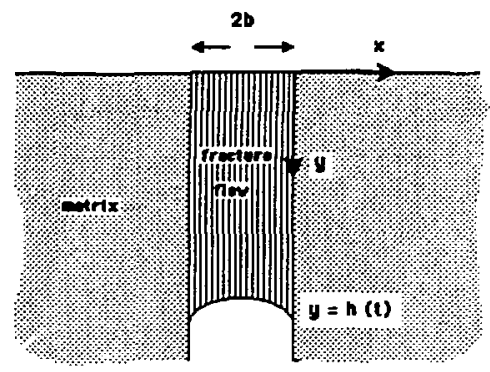

Fizure 2. Fracture Coordinate System 


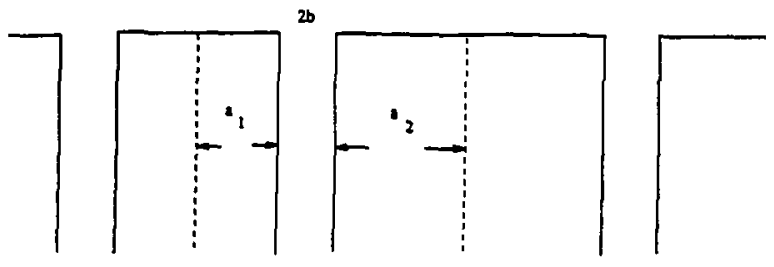

Figure 3. Geometry of Parallel Fracture System

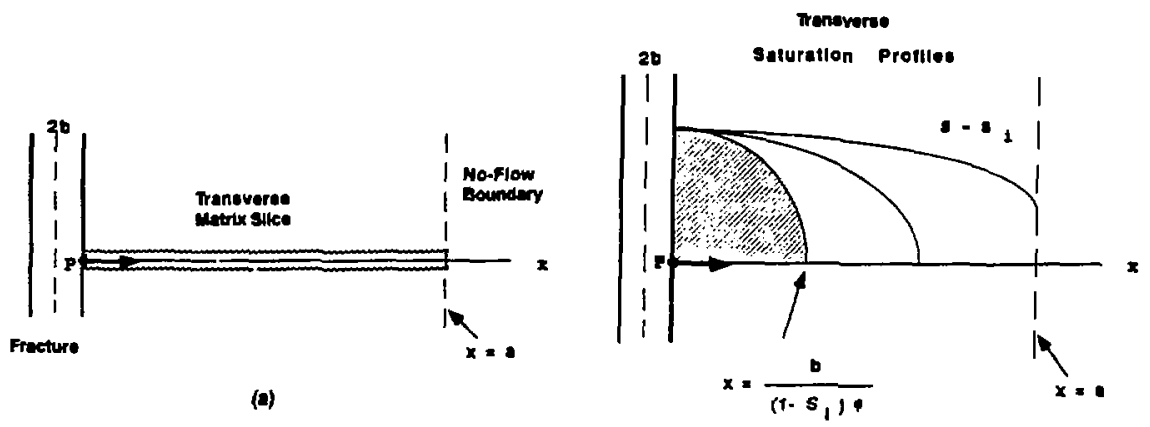

(b)

Figure 4. Stages in Matrix Imbibition 


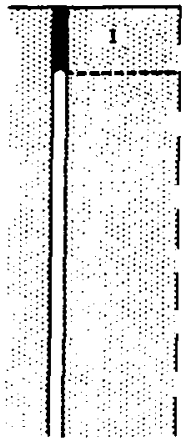

Period 1

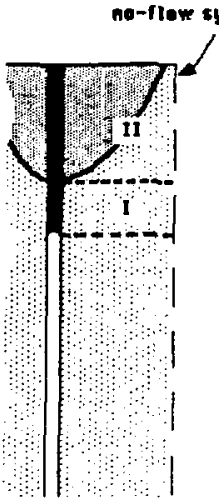

Peried II

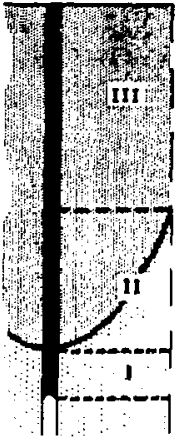

Period III

Figure 5. Flow Periods and Matrix Zones

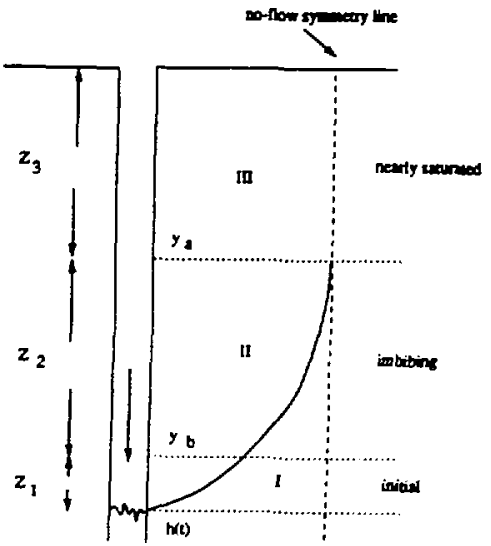

Figure 6. Matrix Zowes 


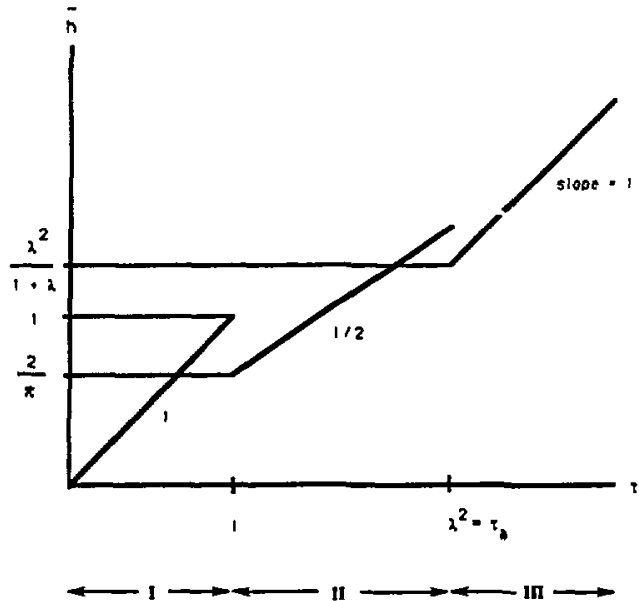

Pigure 7. Asymptotic Profiles for Flux Bouadary Condition (Log-Log)

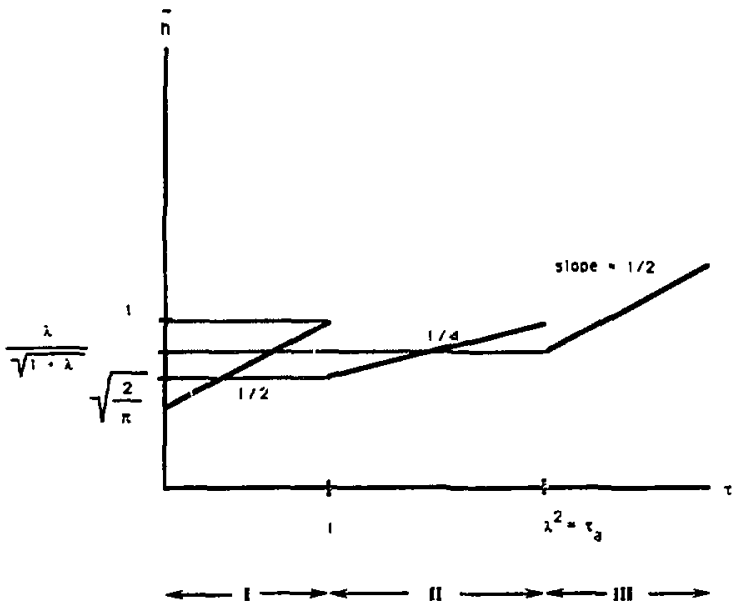

Figure 8. Asymptotic Profiles for Pressure Boundary Condition, No Gravity (Log-Log) 


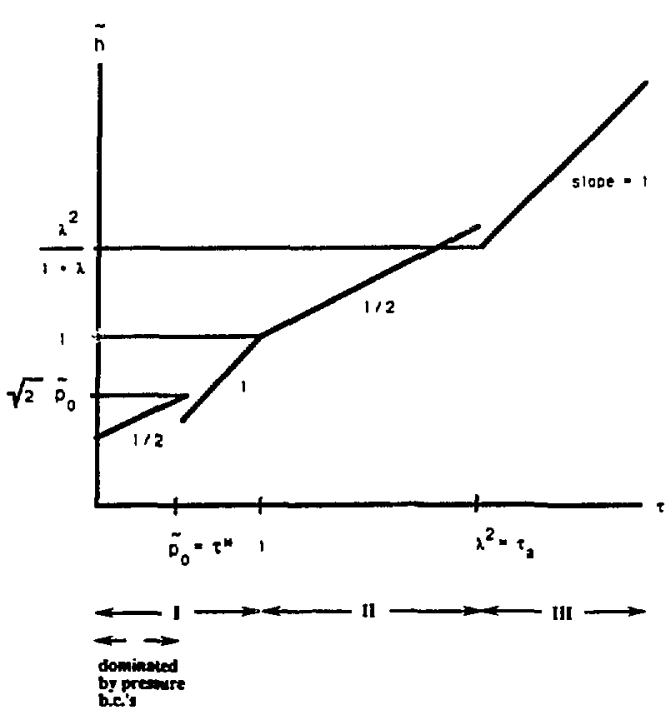

Figure 9. Aysptotic Profiles for Non-Zero Presure Bousdary Condition, $\not_{0} \leq 1$ (Log-Log)

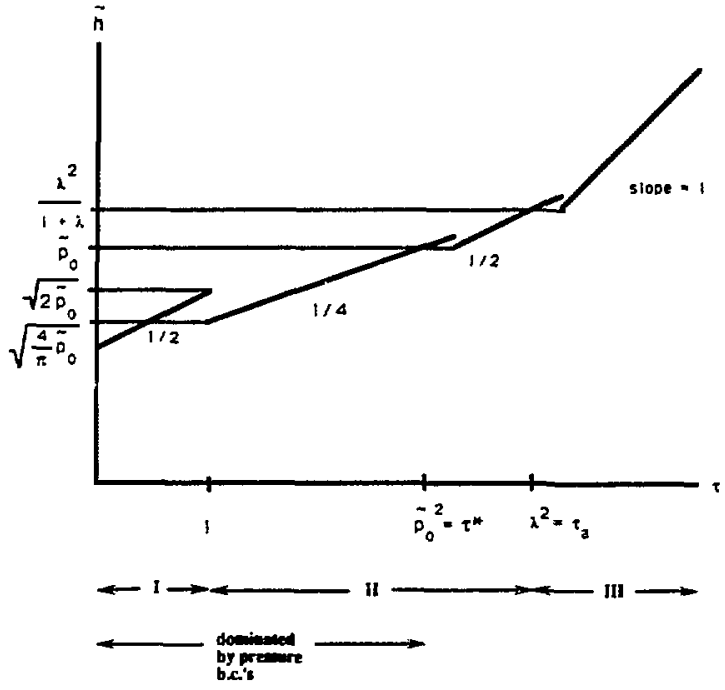

Figure 10. Asymptotic Profiles for Nom-Zero Prescure Bowndary Condition, $1 \leq p_{0} \leq \lambda$ (Log-Log) 


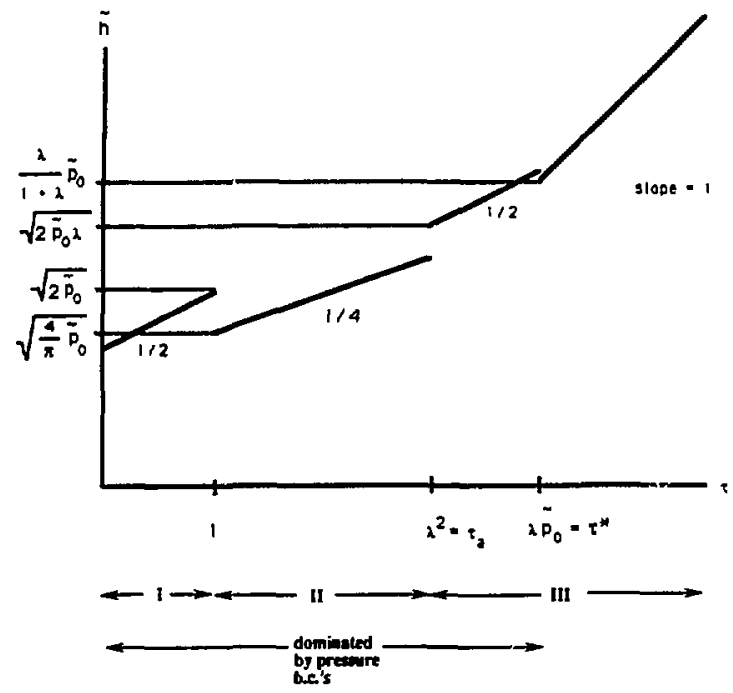

Figure 11. Asymptotic Profiles for Now-Zero Presare Boundary Condition, $\lambda \leq D_{0}$ (Log-Log)

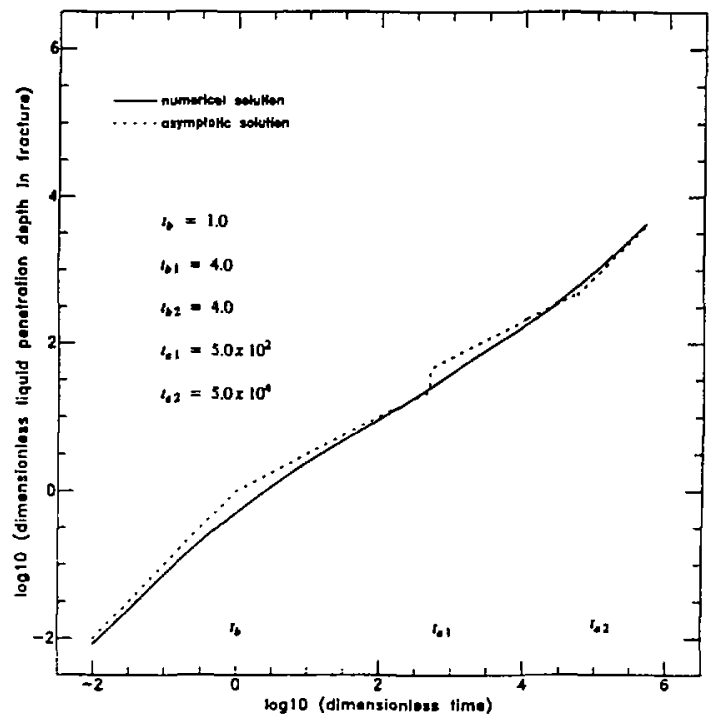

Figure 12. Comparison of First Order Asymptotic Solutions with Numerical Solution to Integro-Differential Equation (zero fracture capillarity, $a_{1} \ll a_{2}$ ) (Log-Log) 


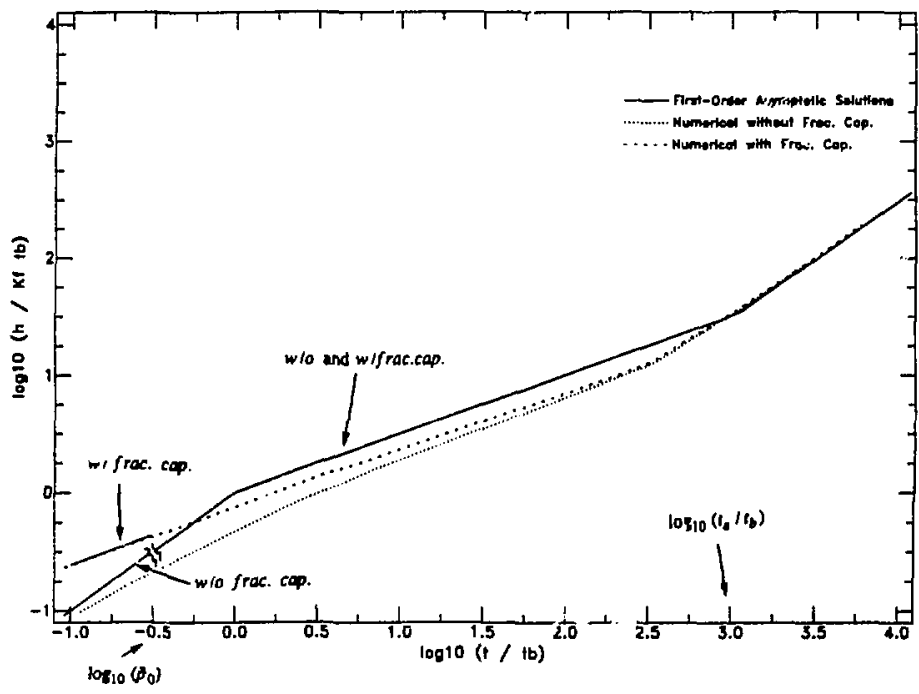

Figure 13. Comparison of First Order Asymptosic Solutions with Two-Dimensional Nomerical Solution (non-zero fracture capillarity, $a_{1}=a_{2}$ ) (Log-Log) 


\section{TABLES}


Table I. Fracture Penetration Length Scale $L_{b}$

\begin{tabular}{c|c} 
Boundary Condition & $L_{b}$ \\
\hline flux b.c. & $\nu_{0} l_{b}$ \\
\hline $\begin{array}{c}\text { pressure b.c } \\
\text { no gravity }\end{array}$ & $\sqrt{2 K_{f} p_{0} \delta_{b}}$ \\
\hline $\begin{array}{c}\text { pressure b.c } \\
\text { with gravity }\end{array}$ & $K_{f} \beta t_{b}$
\end{tabular}

Table I. Leading Term of Expansion for Dimensioniess Penetration $\bar{h}$

\begin{tabular}{|c|c|c|c|c|}
\hline & 1 & 2 & 3 & 4 \\
\hline $\begin{array}{l}\text { flow period } \\
\text { (range of high } \\
\text { accuracy) }\end{array}$ & $\begin{array}{c}\text { flux } \\
\tilde{h}=\frac{h}{x_{0} t_{b}}\end{array}$ & $\begin{array}{c}\text { pressure b.c } \\
\text { no gravity } \\
\bar{h}=\frac{h}{\sqrt{2 p K_{f} l_{b}}}\end{array}$ & $\begin{array}{c}\text { pressure b.c } \\
p_{0}=0, \text { gravity } \\
\tilde{h}=\frac{h}{K_{f} \beta_{b}}\end{array}$ & $\begin{array}{c}\text { pressure b.c } \\
p_{0} \neq 0, \text { gravity } \\
\bar{h}=\frac{h}{K_{f} \beta \ell_{b}}\end{array}$ \\
\hline $\begin{array}{c}I \\
(\tau<<1)\end{array}$ & $\tau$ & $\tau^{1 / 2}$ & $\tau$ & $\begin{array}{c}\tau^{1 / 2} \sqrt{2 p_{0}}, \tau \ll<\bar{p}_{0} \\
\frac{2}{3} \tau, \tau \gg>\bar{p}_{0}>0 \\
\tau, \bar{p}_{0}=0\end{array}$ \\
\hline $\begin{array}{c}\text { II } \\
\left(1<<<<\tau_{d 1}\right)\end{array}$ & $\frac{2}{\pi} \tau^{1 / 2}$ & $\sqrt{\frac{2}{\pi}} \tau^{14}$ & $\tau^{1 / 2}$ & $\begin{array}{c}2 \sqrt{\frac{\overline{\hat{p}}_{0}}{\pi} \tau^{1 \mu}, \tau<<\tilde{p}_{0}^{2}} \\
\tau^{1 / 2}+\frac{\left(2 \bar{p}_{0}-1\right)}{(\pi-2)}, \tau \gg>\tilde{p}_{0}^{2}\end{array}$ \\
\hline $\begin{array}{c}\mathrm{IIa} \\
\left(\tau_{a 1}<<\tau<<\tau_{a z}\right)\end{array}$ & $\frac{2}{\pi}\left(\tau_{2,2} \tau\right)^{1 / 2}$ & $\sqrt{\frac{2}{\pi}}\left(\tau_{1,2} \tau\right)^{14}$ & $\left(\tau_{b 2} \tau\right)^{1 / 2}$ & $\begin{array}{c}2 \sqrt{\frac{\tilde{p}_{0}}{\pi}}\left(\tau_{b 2} \tau\right)^{1 / 4}, \tau<<\tilde{p}_{0}^{2} / \tau_{b 2} \\
\left(\tau_{k 2} \tau\right)^{1 / 2}+\frac{\left(2 \tilde{p}_{0}-\tau_{k 2}\right)}{(\pi-2)}, \tau \gg>\tilde{p}_{0}^{2} / \tau_{b 2}\end{array}$ \\
\hline $\begin{array}{c}I I b \\
\left(\tau_{a 2}<<\tau\right)\end{array}$ & $\frac{1}{1+\lambda} \tau$ & $\frac{1}{\sqrt{1+\lambda}} \tau^{1 / 2}$ & $\frac{1}{1+\lambda} \tau$ & $\begin{array}{c}\sqrt{\frac{2 \bar{p}_{0}}{1+\lambda} \tau^{1 / 2}, \tau<<\tilde{p}_{0}(1+\lambda)} \\
\frac{1}{1+\lambda}\left[\tau+A \tau^{3 / 2}\right], \tau \gg>\tilde{p}_{0}(1+\lambda) \\
A=\left[\frac{2}{3} \frac{\lambda_{1} \tau_{A 1}+\lambda_{2} \tau_{12}}{1+\lambda}+2 \tilde{p}_{0}(1+\lambda)\right]^{1 / 2}\end{array}$ \\
\hline
\end{tabular}


Table III. Leading Term of Expansion for Dimensionless Penetration $\overline{\mathbf{h}}$ (special subcase)

\begin{tabular}{|c|c|c|c|c|}
\hline & 1 & 2 & 3 & 4 \\
\hline $\begin{array}{l}\text { flow period } \\
\text { (range of high } \\
\text { accuracy) }\end{array}$ & $\begin{array}{l}\text { flux } \\
\frac{h}{u_{0} l_{z}}\end{array}$ & $\begin{array}{l}\text { pressure b.c } \\
\text { no gravity } \\
\frac{h}{\sqrt{2 p_{0} K_{f} f_{b}}}\end{array}$ & $\begin{array}{c}\text { pressure b.c } \\
p_{0}=0, \text { gravity } \\
\frac{h}{K_{f} p_{p_{b}}}\end{array}$ & $\begin{array}{c}\text { pressure b.c } \\
p_{0} \neq 0, \text { gravity } \\
\frac{h}{K_{f} \beta_{b}}\end{array}$ \\
\hline $\begin{array}{c}\text { IIIa.1 } \\
\left(\tau_{a},<<\tau<<\tau_{b 2}\right)\end{array}$ & $\frac{1}{1+\lambda_{1}} \tau$ & $\frac{1}{\sqrt{1+\lambda_{1}}} \tau^{1 / 2}$ & $\frac{1}{1+\lambda_{1}} \tau$ & $\begin{array}{c}\frac{1}{1+\lambda_{1}}[\tau+ \\
\left.\left(\tau_{1}, \tau\right)^{1 / 2}\left[\frac{2}{3} \frac{\lambda_{1}^{3 / 2}}{1+\lambda_{1}}+2 \beta_{0}\left(1+\lambda_{1}\right)\right]^{1 / 2}\right)\end{array}$ \\
\hline $\begin{array}{c}\text { IIIa.2 } \\
\left(\tau_{b 2}<<\tau<<\tau_{2}\right)\end{array}$ & $\begin{array}{l}\text { same as for } \\
\text { period IIIa } \\
\text { of Table II }\end{array}$ & $\begin{array}{l}\text { same as for } \\
\text { period IIIa } \\
\text { of Table II }\end{array}$ & $\begin{array}{l}\text { same as for } \\
\text { period IIIa } \\
\text { of Table II }\end{array}$ & $\begin{array}{l}\text { same as for } \\
\text { period IIta } \\
\text { of Table II }\end{array}$ \\
\hline
\end{tabular}

Table IV. Leading Term of Expansion for Dimensionless Peuetration $\tilde{h}$ when $\tau_{a_{1}}=\tau_{a_{2}}$ and $\tau_{b_{1}}=\tau_{b_{2}}$

\begin{tabular}{|c|c|}
\hline $\begin{array}{l}\text { flow period } \\
\text { (range of high } \\
\text { accuracy) }\end{array}$ & $\begin{array}{c}\text { pressure b.c. } \\
\rho_{0} \neq 0, \text { gravity } \\
\tilde{h}=\frac{h}{\bar{K}_{I} \bar{\beta}_{3}}\end{array}$ \\
\hline $\begin{array}{c}\text { I } \\
(\tau<<1)\end{array}$ & $\begin{array}{c}\tau \sqrt{1 / 2} \sqrt{2 \tilde{p}_{0}}, \tau<<\tilde{p}_{0} \\
\frac{2}{3} \tau, \tau>>\tilde{p}_{0} \\
\tau, \tilde{p}_{0}=0\end{array}$ \\
\hline $\begin{array}{c}\text { II } \\
(1<<\tau<<\tau)\end{array}$ & $\begin{array}{c}2 \sqrt{\frac{\bar{p}_{0}}{\pi}} \tau^{14}, \tau \ll \dot{p}_{0}^{2} \\
\tau^{1 / 2}+\frac{\left(2 \tilde{p}_{0}-1\right)}{(\pi-2)}, \tau \gg>\bar{p}_{0}^{2}\end{array}$ \\
\hline $\begin{array}{c}\text { III } \\
\left(\tau_{\mu_{a}}<<\tau\right)\end{array}$ & $\begin{array}{c}\sqrt{\frac{2 \bar{p}_{0}}{1+\lambda}} \tau^{1 / 2}, \tau \ll<\vec{p}_{0}(1+\lambda) \\
\frac{1}{1+\lambda}\left[\tau+A \tau^{1 / 2}\right], \tau \gg>\tilde{p}_{0}(1+\lambda) \\
A=\left[\frac{2}{3} \frac{\lambda^{32}}{1+\lambda}+2 \tilde{p}_{0}(1+\lambda)\right]^{1 / 2}\end{array}$ \\
\hline
\end{tabular}


Table V. Dependence of Fracture Penetration on Parameters $\left(p_{0}=0\right.$, equal matrix properties);

\begin{tabular}{|c|c|c|}
\hline $\begin{array}{l}\text { Flow Period I. } \\
0 \leq 1 \leq t\end{array}$ & $h \sim K_{f}$ & \\
\hline $\begin{array}{l}\text { Flow Period II. } \\
t_{t} \leq t \leq t\end{array}$ & $h \sim \frac{K_{f} b}{S_{m}-S_{i}} \sqrt{\frac{\lambda}{\phi K_{m} \chi}} t^{1}$ & \\
\hline $\begin{array}{l}\text { Flow Period III. } \\
t_{t} \leq t\end{array}$ & $\begin{array}{l}h \sim \frac{K_{f}}{1+a \phi\left(S_{m}-S_{i}\right) / b}, \\
h \sim \frac{K_{f} b}{a \phi\left(S_{m}-S_{l}\right)}{ }^{\prime} \\
h \sim K_{f} l\end{array}$ & $\begin{array}{l}\text { if } a \phi\left(S_{m}-S_{i}\right) \gg b \\
\text { if } a \phi\left(S_{m}-S_{i}\right) \ll b\end{array}$ \\
\hline
\end{tabular}

Table V. Fracture and Mntrix Properties Used in the Two-Dimensional Fracture/Matrix Model

\begin{tabular}{|c|c}
\hline$b$ & $45 \mu \mathrm{m}$ \\
\hline$a$ & $2.26 \mathrm{~cm}$ \\
\hline$K_{f}$ & $8.17 \times 10^{-3} \mathrm{~m} / \mathrm{s}$ \\
\hline$K_{m}$ & $1.86 \times 10^{-11} \mathrm{~m} / \mathrm{s}$ \\
\hline & 20 percent \\
\hline$S_{i}$ & 65 percent \\
\hline$S_{m}$ & 100 percent \\
\hline$l_{b}$ & $41.9 \mathrm{~s}$ \\
\hline$\sigma$ & $3.10 \times 10^{-6} \mathrm{~m}^{2} / \mathrm{s}$ \\
\hline
\end{tabular}

